\title{
A SHORT NOTE ON THE FULL JACOBI GROUP
}

\author{
YOUNGJU CHOIE
}

(Communicated by William Adams)

\begin{abstract}
The full Jacobi group has been defined to study Jacobi forms. The full Jacobi group has properties similar to the modular group. In this note we investigate properties of the full Jacobi group to get generators and relations.
\end{abstract}

\section{INTRODUCTION}

Recently, there have been many studies on the Jacobi forms. Jacobi forms are a mixture of modular forms and elliptic functions. Even though the classical examples are given as the Jacobi theta functions and the Fourier coefficients of Siegel modular forms of genus two, systematic studies have been developed only recently. They are closely related to half integral weight modular form, period of modular form, Heegner points, etc. [see, for example, [1]).

The full Jacobi group has been defined to study Jacobi forms. The full Jacobi group has properties similar to the modular group. In this note we investigate properties of the full Jacobi group to get generators and relations. The relations among the elements in the group will be a useful tool for studying the period of Jacobi forms.

\section{Definitions AND THEOREM}

Let $\Gamma(1)$ be a modular group, that is, a set of 2 by 2 matrices with integer entries and the determinant 1 . The Jacobi group is defined as followings;

Definition 2.1. $\Gamma(1)^{J}:=\Gamma(1) \propto Z^{2}=\left\{[M, X] \mid M \in \Gamma(1), X \in Z^{2}\right\}$. This set $\Gamma(1)^{J}$ forms a group under a group law $[M, X]\left[M^{\prime}, X^{\prime}\right]=\left[M M^{\prime}, X M^{\prime}+X^{\prime}\right]$, and is called the full Jacobi group.

Here, we use the following notation.

Notation 2.1.

$$
T=\left(\begin{array}{cc}
0 & -1 \\
1 & 0
\end{array}\right), \quad S=\left(\begin{array}{ll}
1 & 1 \\
0 & 1
\end{array}\right), \quad P=-T S, \quad I=\left(\begin{array}{ll}
1 & 0 \\
0 & 1
\end{array}\right) .
$$

First of all we state the following well-known lemma without proof (see [3], [4]).

Received by the editors August 25, 1993.

1991 Mathematics Subject Classification. Primary 11F55; Secondary 11F06.

This work was partially supported by KOSEF Research Grant 91-08-00-07, Basic Science N94123 and KOSEF 941-0100-001-2. 
Lemma 2.1. (1) $\Gamma(1)$ is generated by $S$ and $T$. Every element $M \in \Gamma(1)$ can be written in the form

$$
M=S^{q_{0}} T S^{q_{1}} T \cdots T S^{q_{n}}, \quad \text { for } q_{i} \in Z(0 \leq i \leq n) .
$$

(2) Since $S$ and $T$ generate the group $\Gamma(1)$, so do $T$ and $P=-T S$, for $S=T P$.

(3) The generators $T$ and $P$ of the group $\Gamma(1)$ satisfy the relations

$$
T^{4}=P^{3}=I, \quad T^{2} P=P T^{2},
$$

and these are defining relations for $\Gamma(1)$.

We have a similar result on the full Jacobi group. We use the following notation.

Notation 2.2.

$$
\begin{array}{rlrl}
G_{0} & =[S,(0,0)], & G_{1}=[S,(1,0)], & G_{2}=[T,(1,0)], \\
G_{3} & =[I,(1,0)], \quad G_{4}=[I,(0,1)], \quad I^{J}=[I,(0,0)], \\
V & =G_{2}^{3} G_{1}=[-T S,(1,-1)], \quad R=G_{2}^{3} G_{0}=[-T S,(0,-1)] .
\end{array}
$$

Now we state the main theorem.

Theorem 2.1. (1) $\Gamma(1)^{J}$ is generated by $G_{1}$ and $G_{2}$; every element $[M,(\lambda, \mu)]$ $\in \Gamma(1)^{J}$ can be written in the form

$$
[M,(\lambda, \mu)]=G_{1}^{q_{0}} G_{2} G_{1}^{q_{1}} G_{2} \cdots G_{2} G_{1}^{q_{n}},
$$

where $q_{i} \in Z \quad(0 \leq i \leq n)$.

(2) $\Gamma(1)^{J}$ is generated by $G_{0}$ and $G_{2} .[M,(\lambda, \mu)] \in \Gamma(1)^{J}$ can be written in the form

$$
[M,(\lambda, \mu)]=G_{0}^{q_{0}} G_{2} G_{0}^{q_{1}} G_{2} \cdots G_{2} G_{0}^{q_{n}},
$$

where $q_{i} \in Z \quad(0 \leq i \leq n)$.

(3) $\Gamma(1)^{J}$ is generated by $G_{2}$ and $V$. The generators $G_{2}$ and $V$ of the group $\Gamma(1)^{J}$ satisfy the relations

$$
\begin{aligned}
G_{2}^{4} & =V^{3}=I^{J}, \\
V G_{2}^{2} & =[I,(-1,-2)] G_{2}^{2} V=G_{2}^{2}[I,(1,2)] V=G_{2}^{2} V[I,(-2,-1)],
\end{aligned}
$$

and these are defining relations for $\Gamma(1)^{J}$.

(4) $\Gamma(1)^{J}$ is generated by $G_{2}$ and $R$.

Proof of Theorem 2.1. (1) Let $\Gamma_{\text {sub }}^{J}$ be a subgroup generated by $G_{1}, G_{2}, G_{3}$, and $G_{4}$. We first claim that $\Gamma_{\text {sub }}^{J}=\Gamma(1)^{J}$; it is clear that $\Gamma_{\text {sub }}^{J} \subset \Gamma(1)^{J}$. To prove the other direction we have the following from Lemma 2.1:

$$
G_{1}^{q_{0}} G_{2} G_{1}^{q_{1}} G_{2} \cdots G_{2} G_{1}^{q_{n}}=\left[M,\left(\lambda^{\prime}, \mu^{\prime}\right)\right], \quad \text { for }\left(\lambda^{\prime}, \mu^{\prime}\right) \in Z^{2} .
$$

Therefore, for any $[M,(\lambda, \mu)] \in \Gamma(1)^{J}$, there exist integers $\alpha, \beta \in Z$ such that

$$
[M,(\lambda, \mu)]=G_{1}^{q_{0}} G_{2} G_{1}^{q_{1}} G_{2} \ldots G_{2} G_{1}^{q_{n}}[I,(\alpha, \beta)]=\left[M,\left(\lambda^{\prime}, \mu^{\prime}\right)\right] G_{3}^{\alpha} G_{4}^{\beta} .
$$

So we checked that $\Gamma_{\text {sub }}^{J}=\Gamma(1)^{J}$. 
Next we show that $\Gamma_{\text {sub }}^{J}=\Gamma^{J}$, where $\Gamma^{J}$ is generated only by $G_{1}$ and $G_{2}$. We claim that $G_{3}, G_{4} \in \Gamma^{J}$. It is easy to check that

$$
\begin{aligned}
& G_{4}=[I,(0,1)]=\left(G_{2} G_{1}\right)^{2} G_{2}^{3}\left(G_{1} G_{2}^{2}\right)\left(G_{1} G_{2}\right)^{3}, \\
& G_{3}=G_{2}^{3} G_{4} G_{2} .
\end{aligned}
$$

(2) It is sufficient to show that $G_{0}$ and $G_{2}$ generate $G_{1}$ and $G_{2}$. Since $G_{2}^{2} G_{0}=[-S,(1,0)]$ and $G_{2}^{2}\left(G_{0} G_{2}\right)^{3} G_{2}^{2}=[-I,(0,0)]$, we get $G_{1}=[S,(1,0)]$ $=G_{2}^{2}\left(G_{0} G_{2}\right)^{3} G_{0}$. We also note that $G_{0}^{-1} G_{2}^{2}\left(G_{0} G_{2}\right)^{3} G_{0}=G_{3}$. The remaining proof is similar to that of Theorem 2.1(1).

(3) Since $G_{1}$ and $G_{2}$ generate the group $\Gamma(1)^{J}$, so do $G_{2}$ and $V$, for $G_{1}=G_{2} V$. Furthermore, we can easily check that $G_{2}$ and $V$ satisfy the relations (7). Suppose now that an arbitrary relation is given which we assume without restriction to have the form

$$
G_{2}^{\varepsilon_{1}} V^{\delta_{1}} G_{2}^{\varepsilon_{2}} V^{\delta_{2}} \ldots G_{2}^{\varepsilon_{n}} V^{\delta_{n}} G_{2}^{\varepsilon_{n+1}}=I^{J}, \quad \text { where } \varepsilon_{i}, \delta_{j} \in Z .
$$

By applying (7), we can find the unique pair of integers $(\alpha, \beta)$ such that

$$
G_{2}^{\varepsilon_{1}}[I,(\alpha, \beta)] V^{\delta_{1}} G_{2} V^{\delta_{2}} G_{2} \cdots G_{2} V^{\delta_{n}} G_{2}^{\varepsilon_{n}}=I^{J},
$$

where $\varepsilon_{1}, \varepsilon_{n+1}=0,1,2$, or $3, \delta_{i}=1$ or 2 , and $n \geq 0$.

After a suitable multiplication by $G_{2}$ we obtain

$$
[I,(\alpha, \beta)] V^{\delta_{1}} G_{2} V^{\delta_{2}} G_{2} \cdots G_{2} V^{\delta_{n}}=G_{2}^{\delta},
$$

where $\delta_{i}=1$ or $2, \delta=0,1,2$, or 3 , and $n \geq 0$. Then this representation becomes

$$
\begin{aligned}
& {[I,(\alpha, \beta)] V^{\delta_{1}} G_{2} V^{\delta_{2}} G_{2} \cdots G_{2} V^{\delta_{n}}} \\
& \quad=\left[P^{\delta_{1}} T P^{\delta_{2}} T \cdots T P^{\delta_{n}},\left(\alpha^{\prime}, \beta^{\prime}\right)\right] \\
& \quad=\left[T^{\delta},\left(\alpha^{\prime \prime}, \beta^{\prime \prime}\right)\right], \quad \text { for some } \alpha^{\prime}, \beta^{\prime}, \alpha^{\prime \prime}, \beta^{\prime \prime} \in Z .
\end{aligned}
$$

So we have that

$$
P^{\delta_{1}} T P^{\delta_{2}} T \cdots T P^{\delta_{n}}=T^{\delta},
$$

where $\delta_{i}=1$ or $2, \delta=0,1,2$, or 3 , and $n \geq 0$. However, this is not possible for $n \geq 1$ by Lemma 2.1(3). So equation (7) is the only relation in the group $\Gamma(1)^{J}$.

(4) Since $G_{0}=G_{2} R$, the group $\Gamma(1)^{J}$ is generated by $G_{2}$ and $R$.

From the above theorem, we get the following corollary.

Corollary 2.1. (1) The generators $G_{1}$ and $G_{2}$ of the group $\Gamma(1)^{J}$ satisfy the relations

$$
\begin{aligned}
G_{2}^{4} & =\left(G_{2}^{3} G_{1}\right)^{3}=I^{J}, \\
G_{1} G_{2}^{2} & =[I,(2,-1)] G_{2}^{2} G_{1}=G_{2}^{2}[I,(-2,1)] G_{1} \\
& =G_{2}^{2} G_{1}[I,(-2,-1)]
\end{aligned}
$$

and these are defining relations for $\Gamma(1)^{J}$. 
(2) The generators $G_{0}$ and $G_{2}$ of the group $\Gamma(1)^{J}$ satisfy the relations

$$
\begin{aligned}
G_{2}^{4} & =\left(G_{2}^{3} G_{0}\right)^{3}=I^{J}, \\
G_{0} G_{2}^{2} & =[I,(0,1)] G_{2}^{2} G_{0}=G_{2}^{2}[I,(0,-1)] G_{0} \\
& =G_{2}^{2} G_{0}[I,(0,-1)]
\end{aligned}
$$

and these are defining relations for $\Gamma(1)^{J}$.

(3) The generators $R$ and $G_{2}$ of the group $\Gamma(1)^{J}$ satisfy the relations

$$
\begin{aligned}
G_{2}^{4} & =R^{3}=I^{J}, \\
R G_{2}^{2} & =[I,(1,0)] G_{2}^{2} R=G_{2}^{2}[I,(-1,0)] R=G_{2}^{2} R[I,(0,-1)]
\end{aligned}
$$

and these are defining relations for $\Gamma(1)^{J}$.

Proof of Corollary 2.1. (1) Since $V=G_{2}^{3} G_{1}$ and $V^{3}=I^{J}$, we get $\left(G_{2}^{3} G_{1}\right)^{3}=$ $I^{J}$. Also, $G_{1} G_{2}^{2}=G_{2} V G_{2}^{2}=G_{2}[I,(-1,-2)] G_{2}^{2} V=G_{2}[I(-1,-2)] G_{2}^{3} G_{2}^{2} G_{1}=$ $[I,(2,-1)] G_{2}^{2} G_{1}$.

(2) Since $G_{0}=G_{2}^{3} V G_{2}^{3}\left(V G_{2}^{2}\right)^{3} G_{2}=G_{1}[I,(-1,0)]=[I,(-1,1)] G_{1}$, by applying relations on (16), we get (17).

(3) Since $R=G_{2}^{3} G_{0}$, we get (18) by applying (17).

\section{REFERENCES}

1. M. Eichler and D. Zagier, The theory of Jacobi forms, Progr. Math., vol. 55, Birkhäuser, Boston, Basel, and Stuttgart, 1985.

2. M. Newman, Integral matrices, Academic Press, New York, 1972.

3. B. Schoeneberg, Elliptic modular functions, Springer-Verlag, Berlin and New York, 1974.

4. R. Rankin, Modular forms and functions, Cambridge Univ. Press, Cambridge and New York, 1977.

Department of Mathematics, Pohang Institute of Science \& Technology, Pohang, KOREA, 790-784

E-mail address: yjc@vision.postech.ac.kr 